\title{
Video Article \\ Autonomic Function Following Concussion in Youth Athletes: An Exploration of Heart Rate Variability Using 24-hour Recording Methodology
}

\author{
Melissa Paniccia ${ }^{1}$, Tim Taha ${ }^{2}$, Michelle Keightley ${ }^{1,3}$, Scott Thomas ${ }^{2}$, Lee Verweel ${ }^{1}$, James Murphy ${ }^{1}$, Katherine Wilson ${ }^{1}$, Nick Reed ${ }^{1,3,4}$ \\ ${ }^{1}$ Concussion Centre, Bloorview Research Institute, Holland Bloorview Kids Rehabilitation Hospital \\ ${ }^{2}$ Faculty of Kinesiology and Physical Education, University of Toronto \\ ${ }^{3}$ Rehabilitation Sciences Institute, Faculty of Medicine, University of Toronto \\ ${ }^{4}$ Department of Occupational Science and Occupational Therapy, Faculty of Medicine, University of Toronto
}

Correspondence to: Melissa Paniccia at melissa.paniccia@utoronto.ca

URL: https://www.jove.com/video/58203

DOI: doi:10.3791/58203

Keywords: Medicine, Issue 139, Youth, concussion, heart rate variability, 24-hour, autonomic function, non-invasive sensor

Date Published: 9/21/2018

Citation: Paniccia, M., Taha, T., Keightley, M., Thomas, S., Verweel, L., Murphy, J., Wilson, K., Reed, N. Autonomic Function Following Concussion in Youth Athletes: An Exploration of Heart Rate Variability Using 24-hour Recording Methodology. J. Vis. Exp. (139), e58203, doi:10.3791/58203 (2018).

\section{Abstract}

Participation in organized sports makes a significant contribution to youth development, but places youth at a higher risk for sustaining a concussion. To date, return-to-activity decision-making has been anchored in the monitoring of self-reported concussion symptoms and neurocognitive testing. However, multi-modal assessments that corroborate objective physiological measures with traditional subjective symptom reporting are needed and can be valuable. Heart rate variability (HRV) is a non-invasive physiological indicator of the autonomic nervous system, capturing the reciprocal interplay between the sympathetic and parasympathetic nervous systems. There is a dearth of literature exploring the effect of concussion on HRV in youth athletes, and developmental differences preclude the application of adult findings to a pediatric population. Further, the current state of HRV methodology has primarily included short-term (5-15 min) recordings, by using resting state or short-term physical exertion testing to elucidate changes following concussion. The novelty in utilizing a $24 \mathrm{~h}$ recording methodology is that it has the potential to capture natural variation in autonomic function, directly related to the activities a youth athlete performs on a regular basis. Within a prospective, longitudinal research setting, this novel approach to quantifying autonomic function can provide important information regarding the recovery trajectory, alongside traditional self-report symptom measures. Our objectives regarding a $24 \mathrm{~h}$ recording methodology were to (1) evaluate the physiological effects of a concussion in youth athletes, and (2) describe the trajectory of physiological change, while considering the resolution of self-reported post-concussion symptoms. To achieve these objectives, non-invasive sensor technology was implemented. The raw beat-to-beat time intervals captured can be transformed to derive time domain and frequency domain measures, which reflect an individual's ability to adapt and be flexible to their ever-changing environment. By using non-invasive heart rate technology, autonomic function can be quantified outside of a traditional controlled research setting.

\section{Video Link}

The video component of this article can be found at https://www.jove.com/video/58203/

\section{Introduction}

It is estimated that four million children present to emergency departments worldwide with concussion ${ }^{1,2,3}$. The Government of Canada reported that $64 \%$ of emergency department visits among 10-18 year olds are related to physical activity engagement and participation in sports and recreation ${ }^{4}$. Further, $39 \%$ of these aforementioned emergency department visits are comprised of sport-related head injury, and, specifically, concussion. Concussion is a type of traumatic brain injury induced by biomechanical forces, caused by a direct blow to the head or on the body generating force to the head. Concussion results in neurological impairment, which often resolves spontaneously ${ }^{3}$. It is important to highlight that concussion is a functional, rather than structural injury, whereby standard structural imaging studies largely appear unremarkable ${ }^{3}$. Concussion results in the confluence of physical (e.g., headaches, dizziness, blurred vision, and sensitivity to noise and light, decreased strength and motor abilities), cognitive (e.g., difficulty with concentration and recall, slowed thinking and general mental fog), emotional (e.g., sadness, irritability and nervousness) and fatigue (e.g., sleeping too much or too little and feeling drowsy) symptoms ${ }^{5}$.

A major factor in the symptomatology of concussion has been the suspected dysfunction of the autonomic nervous system (ANS) ${ }^{6}$. The ANS is comprised of two branches; the parasympathetic and sympathetic nervous systems. These two branches operate reciprocally to respond to environmental demands with their own idiosyncrasies ${ }^{7}$. The parasympathetic nervous system has less of an influence on peripheral vasculature but is active during periods of energy restoration, allowing the viscera to relax (i.e., decrease heart rate, promote gastrointestinal motility). Conversely, the sympathetic nervous system is activated when stressors are presented and induces a "flight or fight" reaction ${ }^{8}$. To assess the activity of the ANS following concussion, the noninvasive technique of collecting heart rate to quantify heart rate variability (HRV) has been employed $^{8,9,10}$. The sympathetic and parasympathetic nervous systems act on the sinoatrial node (natural electrical pacemaker) of the heart to generate time variations between beat-to-beat (RR) intervals in response to internal and external stressors/stimuli. The beat-to-beat interval 
value across a time series is then used to compute time and frequency domain measures. Time domain variables capture the overall variability of heart rate, while frequency domain variables capture the periodic oscillations of heart rate and provide information on the specific branches of the $\mathrm{ANS}^{8,11,12}$.

The baseline/pre-injury assessment paradigm has typically been employed to quantify changes in the event a youth sustains a concussion. Here, it is standard to obtain a self-report of the youth's concussion symptoms, in addition to other standardized measures such as neuropsychological assessments ${ }^{3}$, in advance of an injury. Following a diagnosed concussion, pre-injury scores are used to make comparisons with post-injury scores to inform recovery and to guide return-to-activity decision making. However, in recent shifts to explore objective indicators of concussion recovery, the value and emphasis on subjective symptom reporting has recently been debated. Youth athletes may not fully understand the consequences of reporting concussion symptoms and may underreport to avoid prolonged absence from sport ${ }^{13,14}$. Further, concussion symptoms are non-specific, and are present across an array of other clinical conditions such as mental health diagnoses ${ }^{15,16}$. The use of HRV within the context of concussion in youth athletes can act to corroborate traditionally used clinical measures (subjective concussion symptoms) and provide novel physiological information (objective HRV) on understanding the post-concussion trajectory.

According to the most recent international consensus statement on concussion in sport, a single "physiological time window" for concussion recovery does not exist due to methodological differences in assessing physiological measures and study design ${ }^{3}$. Multiple studies have suggested physiological dysfunction that outlasts clinical measures of recovery ${ }^{9,17,18}$, but this has not been conclusively established, especially in the youth athlete population. The Task Force of the European Society of Cardiology and the North American Society of Pacing Electrophysiology ${ }^{12}$ provide guidelines on the measurement and analysis of HRV within clinical and research settings. Within the limited concussion literature to date, the vast majority of this novel physiological research has been explored with adult and university-aged athletes in the context of short-term recordings ${ }^{9,18,19}$. However, exploring the use of HRV as an objective measure of recovery within a long-term recording methodology $(24 \mathrm{~h})$, as well as amongst youth athletes, has not been investigated. This is problematic as the factor of ongoing development precludes adult findings from being extrapolated to youth. In line with the Task Force ${ }^{12}$ recommendations and the current limitations in youth concussion literature, there is sufficient rationale to explore the $24 \mathrm{~h}$ recording methodology within youth concussion. It is within the long-term recordings that ANS responses can be evaluated in response to real-world circumstances such as daily normal activities, injury, and the effect of therapeutic interventions, which is key for making predictions on prognosis ${ }^{20}$. Short-term recordings may not accurately represent autonomic modulations in a way that reflects the dynamic fluctuations of natural and expected stressors within the youth's daily activities (i.e., school and physical activities) ${ }^{21}$. Further, the short-term recording has the limitation of not measuring fluctuation in RR intervals, especially given that HRV responds dynamically to physiologic perturbations. Time domain measures, such as SDNN (standard deviation of RR interval), have been used in many studies with 24-hour recording methodology, as they depend on longer-term recordings to reflect accuracy in deriving their value ${ }^{12}$. Given the exploratory nature of this approach to assessing concussion in youth, and the overarching goal of capturing dynamic change, it was suitable to utilize a recording methodology that reflected the daily fluctuation in activity repertoire unique to this pediatric population.

The purpose of this protocol is to explore a non-controlled, $24 \mathrm{~h}$ recording protocol in youth athletes, ages 13-18 years old, participating in normal daily activities. While this $24 \mathrm{~h}$ protocol does introduce variability within the HRV signal, the ability to detect trends despite this variability, may be indicative of a potentially salient signal, and one that may be more ecologically valid ${ }^{20,22}$ when gauging the physiological response to concussive injury in this population.

\section{Protocol}

All steps taken within this protocol were approved in accordance with the Research Ethics Board at Holland Bloorview Kids Rehabilitation Hospital. All participants provided consent prior to their participation in this research protocol/data collection.

\section{Obtain Pre-injury Demographic and Symptom Profile on Participant}

1. Ensure all material is prepared and functioning for data collection (chest strap, sensor, and watch; see Table of Materials) as well as relevant demographic collection, concussion symptom scale, and physical activity forms. Prior to the participant's arrival, remind participant to wear comfortable clothing, which includes a light t-shirt.

2. After obtaining parental and participant consent, request that the participant fill out the demographic collection form, which includes age, sex, diagnosed learning disabilities, other medical diagnoses, and previous history of concussion (number and recency of injuries).

3. Instruct the participant to complete the Godin Leisure-Time Exercise Questionnaire (GLTE) $)^{23}$.

4. Instruct the participant to complete the Post-Concussion Symptom Inventory (PCSI) ${ }^{24}$. NOTE: There are various PCSI versions, which correspond to relevant developmental age ranges (i.e., 5 to 12 year olds; 13 to 18 year olds).

5. Measure and record the participants' height and weight.

6. Score and record the GLTE and PCSI. When scoring the PCSI, record both the total score as well as the four domain scores (i.e., physical, cognitive, emotional, fatigue).

7. Enter all demographic, physical activity and symptom scale information into a database.

\section{Obtain Pre-injury Physiological Profile on Participant}

NOTE: It is important to note that delineating a weekday versus a weekend or specific time of day for data collection is not always feasible given the busy school and sport schedule of the youth athlete. Protocols should take every effort to ensure consistency, or account for time of day in data analyses to address the natural variations in autonomic nervous system function. Further, if a participant sustains a concussion, but their baseline value was collected more than one year following the date of their concussion, the baseline value should not be considered accurate for the purposes of pre-post comparisons ${ }^{25}$.

1. Select appropriate chest strap size (XS/S or M/XXL), according to circumference of participant's torso. 
1. To ensure accurate chest strap placement, place the strap around the torso of the participant (note: while participant is clothed with a tshirt) and adjust the strap to reflect a tight, yet comfortable fit.

2. Ensure the strap is securely fitted about the sternum, on the xiphoid process. Do not instruct the participant to place the strap directly onto their skin at this time.

2. Connect the heart rate sensor to the chest strap by securing the clip-on buttons, ensuring the sensor is right side up.

3. Using hypoallergenic electrode gel, apply a small/modest amount of gel to the conductive plastic surface of the chest strap.

4. Provide instructions to the participant to access a private area or washroom so the participant can place the chest strap directly onto their skin.

1. Orient the participant to the chest strap clasp to ensure ease in securing the strap.

2. Instruct the participant to ensure that the sensor should be placed directly onto the xiphoid process of the sternum and right side up to ensure optimal heart rate recording.

5. Provide the participant with the watch, instructing them not to remove the watch throughout the $24 \mathrm{~h}$ recording duration.

6. Provide the participant with a trouble-shooting instruction sheet (in the event the watch accidently stops recording). The troubleshooting instruction sheet should explicitly outline how to re-start the watch recording and how to re-apply the heart rate monitor with electrode gel, adjust the strap, and ensure the sensor is right side up. Given the length of recording time and the potential for inconsistent recording length across participants, use rigorous data analysis methods to filter and account for this discrepancy; please see Paniccia et al. $^{26}$ for further details on these approaches.

1. Include an area on this instruction sheet for the participant to record when they went to sleep and when they woke up. Also, encourage participants to report on other physical activities they engage in while wearing the heart rate equipment (e.g., going for a long walk).

7. Remind the participant that participation in contact sport, swimming, and bathing are not permitted while wearing the heart rate technology. However, the participant is permitted to shower with these items on.

1. Encourage the participant to go about their typical activities as they would on a daily basis.

\section{Performing Post-concussion Follow-up Assessment}

1. Schedule a follow-up assessment on the same day that the participant or legal guardian/caregiver indicates a concussive injury (or as soon as possible). Instruct the participant to receive a concussion diagnosis from a physician if they haven't already done so.

2. Ensure all material is prepared and functioning for data collection; this includes the heart rate recording equipment (chest strap, sensor, and watch) and relevant demographic collection, symptom scale, and physical activity forms. Prior to the participant's arrival, remind participant to wear comfortable clothing, which includes a light t-shirt.

3. Complete the Acute Concussion Evaluation form ${ }^{5}$ to collect information on the mechanism of injury and post-injury sequelae (e.g., loss of consciousness, retrograde amnesia).

4. Administer the GLTE to capture change in physical activity repertoire.

5. Administer the PCSI to determine number and severity of symptoms.

6. Collect HRV data and repeat steps 2.1 to 2.7 .

7. While the participant is symptomatic, schedule weekly follow-up assessments and re-administer PCSI and collect HRV data (steps 3.3 and steps 2.1 to 2.7 ).

8. When the participant's symptoms have abated (returned to baseline level), schedule 1-, 3- and 6-month follow-up assessments. At these time points, re-administer GLTE, PCSI and collect HRV data (steps 3.4 and 3.5, and steps 2.1 to 2.7).

\section{Collect Matched Control Participant Data Across Follow-up Assessment Time Points}

1. Utilizing the baseline/pre-injury dataset, generate a list of potential participants, age and gender matched to the concussed participants. Ensure the birthdate of the potential control participant is within 6 months of the concussed participant.

2. Contact control participants and schedule follow-up assessments, with no more than 3-4 days from the concussed participant follow-up date.

3. Replicate above testing protocol to the matched control (i.e., same number of follow-up assessments).

1. On the first follow-up assessment, repeat steps 1.1 to 1.7 and steps 2.1 to 2.7 .

2. On subsequent follow-up assessments, repeat steps 3.1 to 3.7 (excluding step 3.3 ).

\section{Uploading and Processing HRV Data}

1. Connect watch to a computer with the provided USB Transfer cable and upload the heart rate data to the software program provided with the sensor. Next, transfer this specific (.hrm) file and upload the data to the data analysis software (see Table of Materials).

2. To filter ectopic beats and correct artifacts, select the "very low" filter; this is the lowest correction level to maintain the integrity of the raw data. Visual inspection of data files is highly recommended for small datasets and within-participant analyses.

3. In line with recommendations from the Task Force of the European Society of Cardiology and the North American Society of Pacing and Electrophysiology ${ }^{12}$, ensure the frequency domain variable bandwidths are selected as follows: $\mathrm{HF}(0.15$ to $0.4 \mathrm{~Hz})$; LF (0.04 to $0.15 \mathrm{~Hz}$ ).

1. Select a $300 \mathrm{~s}$ window frame, $50 \%$ overlap.

2. Select an interpolation rate of $4 \mathrm{~Hz}$.

3. Select the Fast Fourier Transform for power spectral analysis.

4. Save the HRV data as an hrm file for potential data analysis in robust statistical software. 


\section{Representative Results}

\section{RS800CX Technology Set-up}

Using the procedure presented here, it is important to highlight that the placement and security of the chest strap is crucial to prevent the strap from falling or moving excessively over the $24 \mathrm{~h}$ recording period and consequently, in the collection of an accurate recording. Figure 1 depicts the optimal placement of the heart rate technology, noting that the chest strap and sensor are secure and centered on the youth's sternum (xiphoid process).

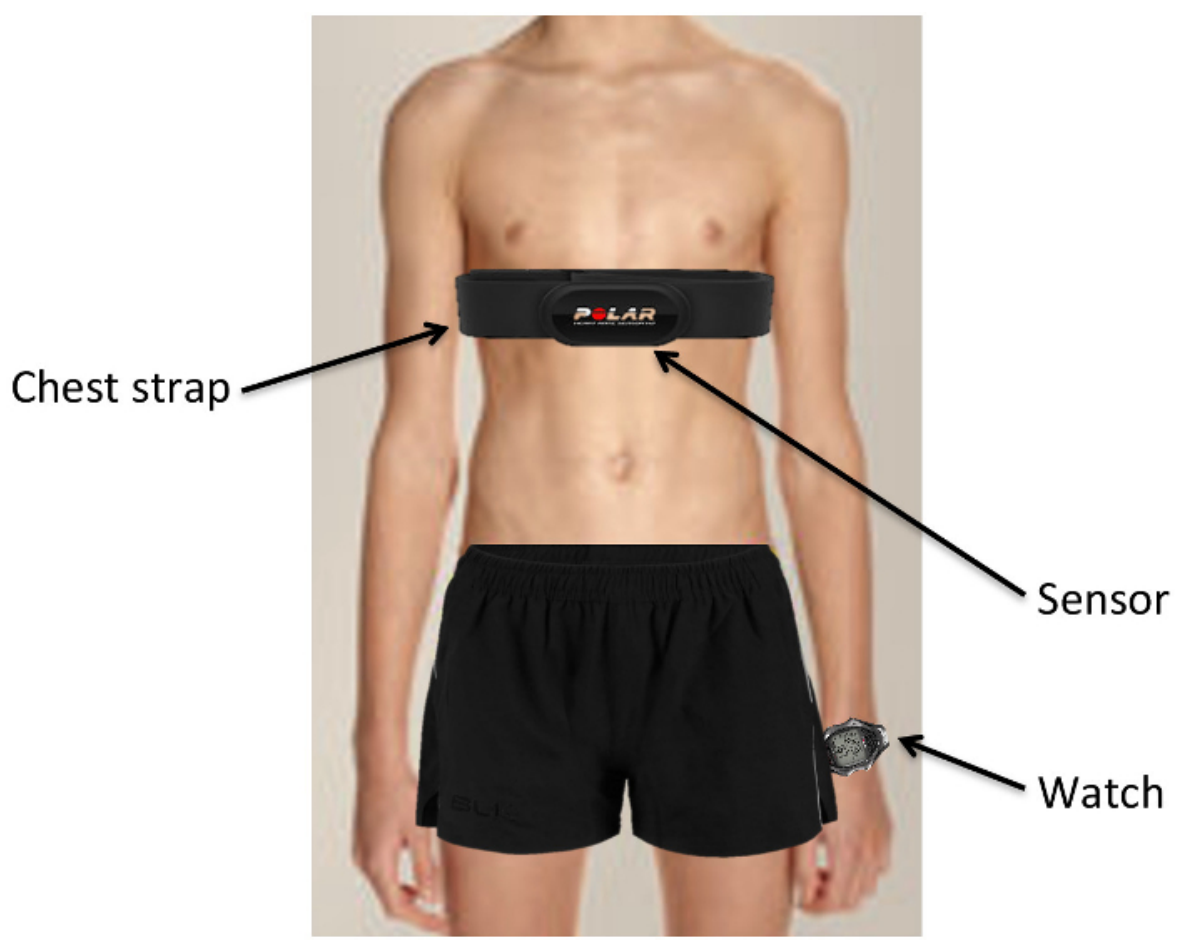

Figure 1: Diagram of heart rate technology, depicting optimal placement of chest strap, sensor and watch. Please click here to view a larger version of this figure.

\section{Heart Rate Variability Output Data}

Figure 2 depicts the Kubios output of a $24 \mathrm{~h}$ heart rate recording for a concussed participant. The image of the raw RR series allows the researcher to visualize change across time, highlighting key time points of increase (e.g., physical activity) or decrease (e.g., resting, sleeping) that are important for the interpretation of data. Time and frequency domain variables represent the overall variability of the physiological signal and the branches of ANS, respectively. Table 1 provides an overview of the units of measurement for time and frequency domain HRV variables and their physiological significance. 

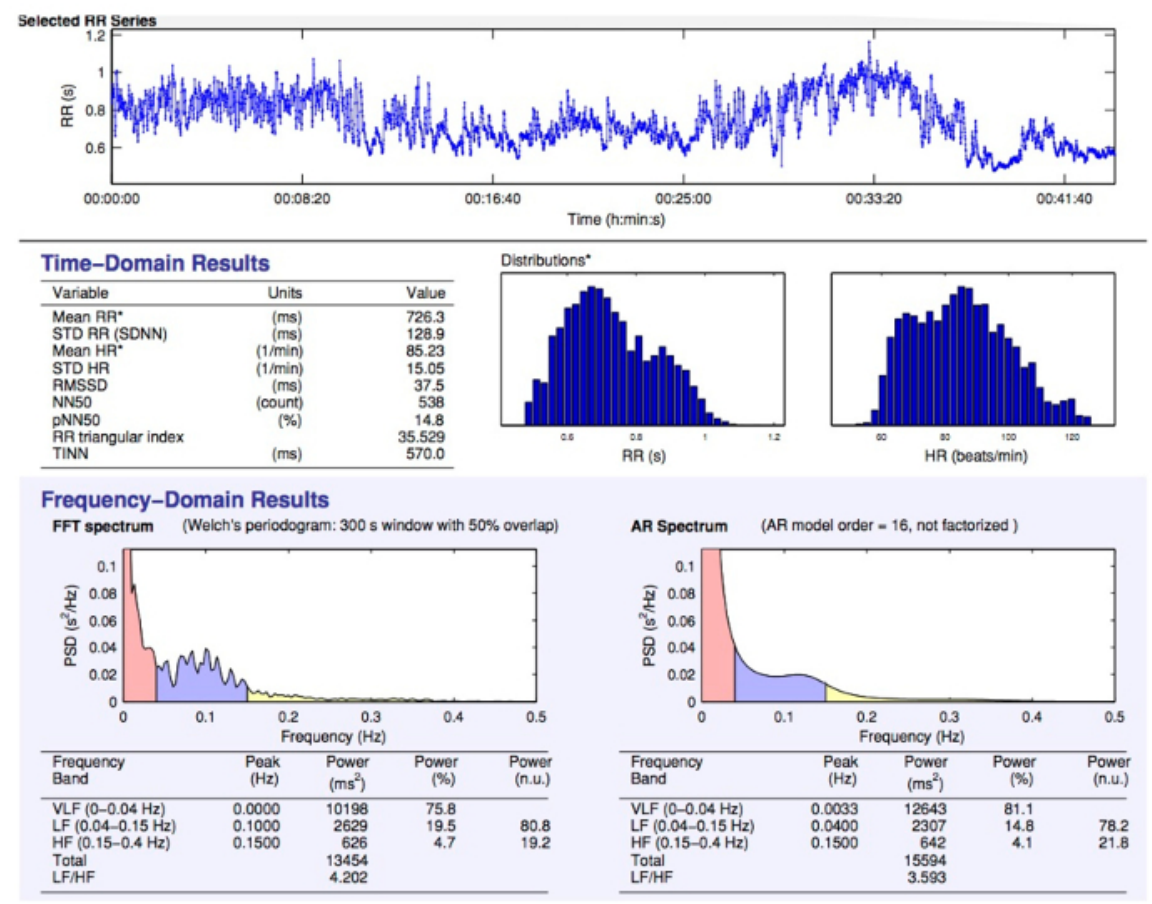

Figure 2: Sample $24 \mathrm{~h}$ data output depicting raw RR series, time domain variables and frequency domain variables. Please click here to view a larger version of this figure. 


\begin{tabular}{|c|c|c|c|}
\hline Variable (units) & Definition & Physiological Significance & \\
\hline \multicolumn{4}{|l|}{ Time Domain } \\
\hline SDNN (ms) & $\begin{array}{l}\text { Standard deviation of intervals } \\
\text { between heartbeats }\end{array}$ & Global index of ANS function & \\
\hline RMSSD (ms) & $\begin{array}{l}\text { Root mean square of successive } \\
\text { differences; calculated through } \\
\text { squaring the intervals between } \\
\text { heartbeats }\end{array}$ & Global index of ANS function & \\
\hline pNN50 (\%) & $\begin{array}{l}\text { Proportion of heartbeat intervals } \\
\text { that differ by more than } 50 \mathrm{~ms}\end{array}$ & $\begin{array}{l}\text { Indicative of parasympathetic } \\
\text { activity }\end{array}$ & \\
\hline \multirow[t]{2}{*}{ NN50 } & A count variable; & \multirow{2}{*}{$\begin{array}{l}\text { Indicative of parasympathetic } \\
\text { activity }\end{array}$} & \\
\hline & $\begin{array}{l}\text { number of pairs of adjacent } \mathrm{NN} \\
\text { intervals differing by more than } 50 \\
\text { ms }\end{array}$ & & \\
\hline STD HR (s) & $\begin{array}{l}\text { Standard deviation of } \\
\text { instantaneous heart rate values }\end{array}$ & Global index of ANS function & \\
\hline \multicolumn{4}{|l|}{ Geometric Methods } \\
\hline \multirow[t]{2}{*}{ RR Triangular Index } & $\begin{array}{l}\text { Total number of all NN intervals } \\
\text { divided by the height of the } \\
\text { histogram of all NN intervals }\end{array}$ & \multirow[t]{2}{*}{ Global index of ANS function } & \\
\hline & $\begin{array}{l}\text { measured on a discrete scale (i.e. } \\
\text { the number of all NN intervals } \\
\text { divided by the maximum of the } \\
\text { density distribution) }\end{array}$ & & \\
\hline TINN (ms) & $\begin{array}{l}\text { Baseline width of the distribution } \\
\text { measured as a base of a triangle, } \\
\text { approximating the NN interval } \\
\text { distribution }\end{array}$ & Global index of ANS function & \\
\hline \multicolumn{3}{|l|}{ Frequency Domain } & \\
\hline $\mathrm{HF}\left(\mathrm{ms}^{2}\right)$ & $\begin{array}{l}\text { Power (magnitude) in high } \\
\text { frequency range, } 0.15-0.4 \mathrm{~Hz}\end{array}$ & $\begin{array}{l}\text { Index of parasympathetic activity } \\
\text { on heart based on rhythmic } \\
\text { respiration cycles }\end{array}$ & \\
\hline HFnu (\%) & $\begin{array}{l}\text { HF power in normalized units, as } \\
\text { a ratio of the total power; [HF/(HF } \\
+\mathrm{LF})] \times 100\end{array}$ & $\begin{array}{l}\text { Proportion of parasympathetic } \\
\text { activity }\end{array}$ & \\
\hline $\operatorname{LF}\left(\mathrm{ms}^{2}\right)^{*}$ & $\begin{array}{l}\text { Power (magnitude) in low } \\
\text { frequency range, } 0.04-0.15 \mathrm{~Hz}\end{array}$ & $\begin{array}{l}\text { Measure of sympathetic and/or } \\
\text { parasympathetic activity }\end{array}$ & \\
\hline LFnu $(\%)^{*}$ & $\begin{array}{l}\text { LF power in normalized units, as } \\
\text { a ratio of the total power; [LF/(HF } \\
+\mathrm{LF})] \times 100\end{array}$ & $\begin{array}{l}\text { Measure of sympathetic and/or } \\
\text { parasympathetic activity }\end{array}$ & \\
\hline $\mathrm{LF} / \mathrm{HF}\left(\mathrm{ms}^{2}\right)^{*}$ & $\begin{array}{l}\text { Ratio of low frequency power to } \\
\text { high frequency power }\end{array}$ & $\begin{array}{l}\text { Measure of sympathetic and/or } \\
\text { parasympathetic activity }\end{array}$ & \\
\hline Total power $\left(\mathrm{ms}^{2}\right)$ & Variance of all RR intervals & $\begin{array}{l}\text { Overall magnitude of variability } \\
\text { within ANS; ability of ANS system } \\
\text { to be flexible and adaptable }\end{array}$ & \\
\hline
\end{tabular}

Table 1: Description of time domain and frequency domain HRV variables.

NOTE: "*" denotes cautionary interpretation of LF-related variables, as the clinical usefulness and validity of this measure is controversial. It has been postulated that LF is not representative of sympathetic autonomic modulation ${ }^{27}$, in addition to having a poor relationship to sympathetic nerve activation ${ }^{28}$. Thus, it is challenging to decipher the physiological basis of this measure.

\section{Visualizing Subjective and Objective Findings}


Given the novelty in $24 \mathrm{~h}$ recording methodology, visualization of findings across the recovery trajectory is key to interpretation of findings with respect to mapping "clinical recovery" with potential "physiological recovery". It is important to note that consistency is maintained regarding the investigator that interprets visual trends in order to ensure reliability. In this particular protocol, the primary author, well versed in neurophysiology, interpreted all aggregate trends. The interpretation of these trends was then reviewed within an interdisciplinary context of experts in pediatric concussion, theoretical physiology and exercise physiology. Thus, a well-informed approach to visualizations is key to making preliminary inferences regarding the recovery trajectory. Figure 3 depicts the relationship between pNN50 and PCSI total score across days post-injury, stratified by sex. Within a longitudinal research setting, collecting repeated measures data could provide rich HRV data. Figure 3 is an example of the recovery trajectory when comparing youth males to youth females. Here, the recovery trajectory appears to be similar within both males (A) and females (B), whereby an initial decrease is found until day 30, followed by increases until day 75/90 for males and females respectively, and then followed by a plateau.

\section{(A) Male recovery trajectory}

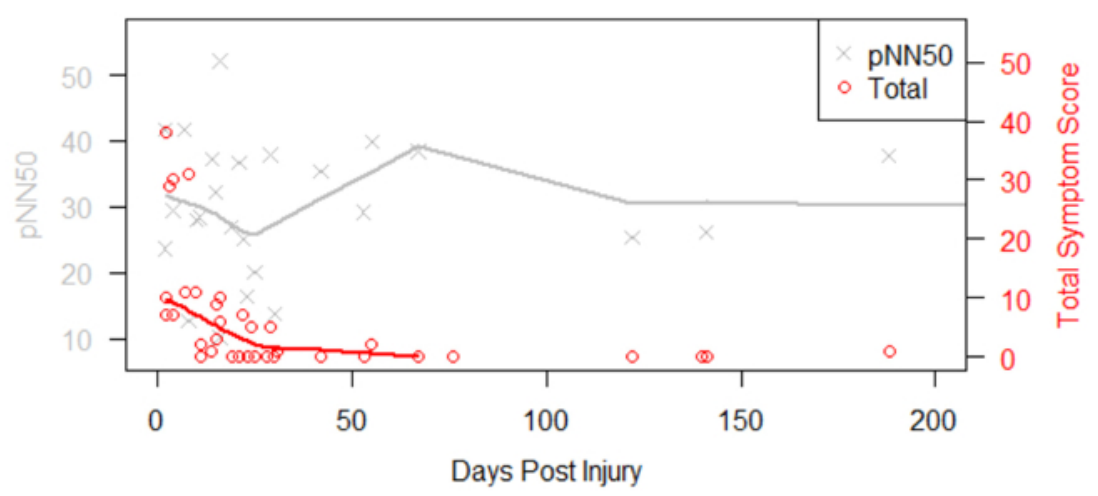

\section{(B) Female recovery trajectory}

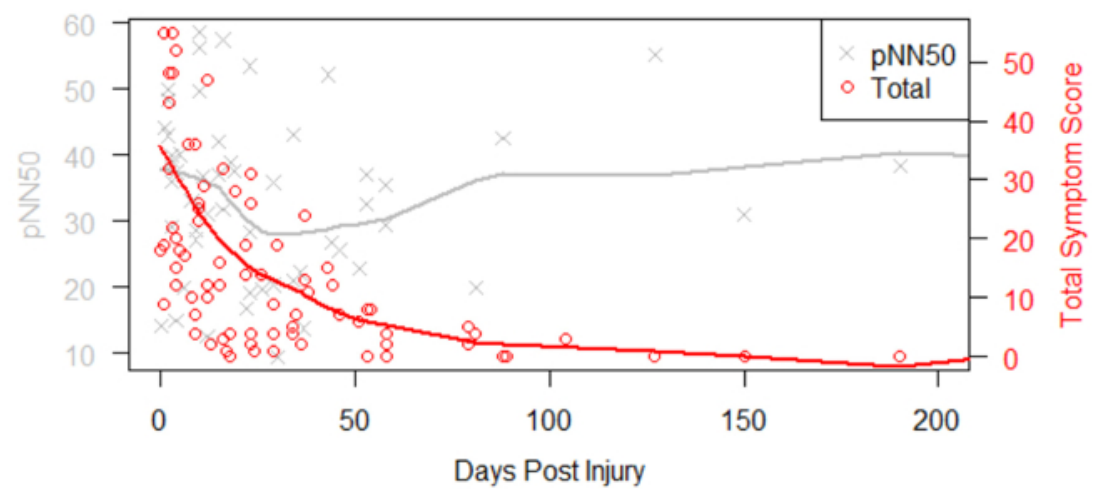

Figure 3: Visualization of recovery trajectory across days post injury, stratified by sex. Please note that "Total" in the legend box reflects the total PCSI score. Please click here to view a larger version of this figure.

\section{Discussion}

Clinical research has indicated that the assessment of the athlete should not simply be comprised of a catalogue of concussion symptoms ${ }^{17}$. Thus, objective physiological measures such as HRV can be of value to disentangle the many factors that play a role in subjective symptom reporting and to elucidate a physiological recovery trajectory that may present differently from a clinical recovery trajectory. This protocol acts as a first step in exploring the role of $24 \mathrm{~h}$ recording methodology in a youth concussion research setting, while accounting for significant pre-injury $\mathrm{HRV}$ and the follow-up of youth athletes (those with concussion and controls) across multiple time points.

The assessment of concussion has been traditionally anchored in the evaluation and resolution of self-reported symptoms. However, there are barriers to this traditional model, which limits the research and clinical community's ability to utilize more objective approaches to assess recovery. For example, if a youth is experiencing symptoms during an assessment following concussion and is unable to complete follow-up testing, the assessment is typically terminated with the inability to gauge how their performance was affected compared to their baseline ${ }^{29}$. Further, a youth's symptom score does not necessarily correlate to their ability to carry out daily activities; a youth may report a reduction in their post-concussion symptoms but experience symptom exacerbation (e.g. mental fog, difficulty concentrating, fatigue, headache) when returning to a full school day ${ }^{29,30,31}$ or when attempting to return to sport or physical activity too soon ${ }^{32,33}$. It may be that more objective measures can capture the sensitivities related to continued recovery, despite the clinical recovery of symptoms. Taken together, the exploration of a non- 
invasive and objective measure can promote the discovery and use of a safe and more sensitive measure of recovery for youth athletes following a concussion.

The present protocol proposes the value in utilizing a novel objective indicator of recovery (i.e., long term recording of HRV). However, autonomic nervous system function can also be impacted by an individual's mental health status and their ability to self-regulate, in the context of stress ${ }^{34,35}$ Not captured in this study is the possibility that changes in HRV following concussion may result from secondary mental health issues that arise when youth are restricted from engaging in meaningful activity ${ }^{30}$ and subsequently, slow to recover from concussion. Indeed, previous work has revealed the relationship between reductions in HRV in young people with non-clinical and clinical forms of depression and anxiety ${ }^{15,16}$. Thus, future study should elaborate on the current protocol to include measures that capture both pre-injury baseline mental health status and how this status may or may not change following concussion.

Kamins et al. ${ }^{36}$ conducted a systematic review, highlighting that the scope of physiological recovery following concussion included physical activity, balance, cognition and oculomotor dysfunction. While this 24-hour recording approach has been deemed an ecologically valid approach to assessing change in autonomic function ${ }^{37}$, the frequency and intensity of physical activity can limit the extent to which changes in HRV can be interpreted. Levels of physical activity likely differ across age and this can contribute to large variances seen within HRV variables. Objectively capturing and quantifying physical activity was not presented within this protocol; thus, it is possible that some youth athletes were in a competitive phase, training phase or even off-season. Research has demonstrated a significant association between physical activity and

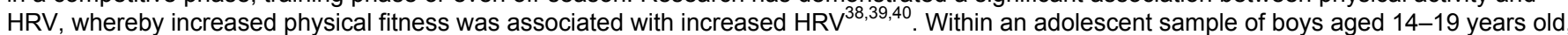
higher reported levels of physical activity was positively associated with both time and frequency domain HRV measures ${ }^{41}$. To address this gap, future exploration could utilize actigraphy technology to capture the type, frequency, and intensity of physical activity, while concurrently collecting HRV. Equally important to consider is the role of cognitive demand/activity in everyday life (i.e., academic demands, extracurricular activities). While this study did not collect information in the form of a cognitive diary or employ other forms of cognitive activity tracking, it is important to consider these clinical measures to allow a fulsome interpretation of the autonomic nervous system. Finally, while oculomotor function was not captured in the current protocol, Hunt et al. ${ }^{42}$ found that measurements of saccades, smooth pursuit, and vergence may be useful in detecting changes associated with concussion. Clinicians and researchers are directed to the broader multi-modal assessment protocol published by Reed et al. ${ }^{29}$ for information on balance and cognitive assessment following concussive injury.

The challenge in the present protocol, while it does provide rich physiological data, is the inability to directly compare results to other similar concussion studies. The majority of HRV concussion studies have employed a resting state position protocol ${ }^{9,19}$ or observe changes in HRV according to exercise exertion ${ }^{43,44,45}$. The $24 \mathrm{~h}$ recording protocol presented here was open-ended in that youth were instructed to carry out their normal daily routines. Additionally, while youth were instructed to document their sleep and wake times, subjective reporting does not provide a clear indication of sleep quality. Sleep factors can significantly impact how an individual carries out their daily activities and can serve as a feedback loop to the ANS to regulate stress responses ${ }^{46}$. Thus, a standardized assessment (i.e., sleep questionnaire and actigraphy) to quantify these sleep factors is needed for more robust interpretations of this $24 \mathrm{~h}$ recording.

A final consideration for the current protocol is the impact of menstruation on HRV when considering pre- and post-concussion comparisons. While this factor was not collected within the present protocol, the potential influence of menstruation is important to account for as studies have shown that autonomic nervous system function fluctuates during the menstrual cycle, in which the alteration of ovarian hormones might be responsible for changes seen in HRV ${ }^{47,48}$. However, it is unclear how the menstrual cycle may or may not have played a role in symptom reporting or in HRV changes within this study sample of 13-18 year olds. Capturing developmental stage in addition to gauging the phase of the menstrual cycle will likely provide additional information on elucidating potential changes following concussion.

In summary, this protocol does provide a clinically relevant means to evaluate change within an objective physiological measure, in an understudied population. Going forward, it will be important to also explore the role of the factors presented above, along with perceived everyday stress and personal athlete characteristics, to decipher if concussion impacts the ANS above and beyond what would be expected from a stressful life event.

\section{Disclosures}

No conflicts of interest declared.

\section{Acknowledgements}

This is work was funded by the Canadian Institutes of Health Research (\#127048), the Ontario Neurotrauma Foundation and the Ontario Brain Institute. The Ontario Brain Institute is an inde- pendent non-profit corporation, funded partially by the Ontario government. The opinions, results, and conclusions are those of the authors and no endorsement by the Ontario Brain Institute is intended or should be inferred. This work was originally featured within the first author's doctoral thesis, as part of a larger body of work on neurophysiological variation (Paniccia, 2018, [University of Toronto, unpublished doctoral thesis]). The authors thank the Rehabilitation Sciences Institute at the University of Toronto for their support throughout the doctoral program. We would also like to acknowledge the efforts of the members of the CIHR "NeuroCare" Team and the members of the Concussion Centre (Bloorview Research Institute), specifically Talia Dick, and Katherine Mah. We are grateful to the youth and families for their participation in this research.

\section{References}

1. Aubry, M. et al. Summary and agreement statement of the First International Conference on Concussion in Sport, Vienna 2001. Recommendations for the improvement of safety and health of athletes who may suffer concussive injuries. British Journal of Sports Medicine. 36 (1), 6-10 (2002). 
2. McCrory, P. Summary and agreement statement of the 2nd International Conference on Concussion in Sport, Prague 2004. British Journal of Sports Medicine. 39 (Supplement 1), i78-i86 (2005).

3. McCrory, P. et al. Consensus statement on concussion in sport-the $5^{\text {th }}$ international conference on concussion in sport held in Berlin, October 2016. British Journal of Sports Medicine. (2017).

4. Government of Canada. Concussions. at <http://canada.pch.gc.ca/eng/1465244566173>. (2017).

5. Gioia, G., Collins, M. Acute Concussion Evaluation. <https://www.cdc.gov/headsup/pdfs/providers/ace-a.pdf> (2006).

6. Leddy, J.J., Kozlowski, K., Fung, M., Pendergast, D.R., Willer, B. Regulatory and autoregulatory physiological dysfunction as a primary characteristic of post concussion syndrome: implications for treatment. NeuroRehabilitation. 22 (3), 199-205 (2007).

7. Leddy, J.J., Kozlowski, K., Fung, M., Pendergast, D.R., Willer, B. Regulatory and autoregulatory physiological dysfunction as a primary characteristic of post concussion syndrome: implications for treatment. NeuroRehabilitation. 22 (3), 199-205 (2007).

8. Esterov, D., Greenwald, B. Autonomic Dysfunction after Mild Traumatic Brain Injury. Brain Sciences. 7 (8), 100 (2017).

9. Hutchison, M.G., Mainwaring, L., Senthinathan, A., Churchill, N., Thomas, S., Richards, D. Psychological and Physiological Markers of Stress in Concussed Athletes Across Recovery Milestones. The Journal of Head Trauma Rehabilitation. 32 (3), E38-E48 (2017).

10. Willer, B., Leddy, J.J. Management of concussion and post-concussion syndrome. Current Treatment Options in Neurology. 8 (5), $415-426$ (2006).

11. Aubert, A.E., Seps, B., Beckers, F. Heart rate variability in athletes. Sports Medicine (Auckland, N.Z.). 33 (12), $889-919$ (2003).

12. Task Force of the European Society of Cardiology and the North American Society of Pacing and Electrophysiology. Heart rate variability: standards of measurement, physiological interpretation and clinical use. Circulation. 93 (5), 1043-1065 (1996).

13. Bailey, C.M., Echemendia, R.J., Arnett, P.A. The impact of motivation on neuropsychological performance in sports-related mild traumatic brain injury. Journal of the International Neuropsychological Society: JINS. 12 (4), 475-484 (2006).

14. Echemendia, R.J., Putukian, M., Mackin, R.S., Julian, L., Shoss, N. Neuropsychological test performance prior to and following sports-related mild traumatic brain injury. Clinical Journal of Sport Medicine: Official Journal of the Canadian Academy of Sport Medicine. 11 (1), $23-31$ (2001).

15. Koenig, J., Kemp, A.H., Beauchaine, T.P., Thayer, J.F., Kaess, M. Depression and resting state heart rate variability in children and adolescents - A systematic review and meta-analysis. Clinical Psychology Review. 46, 136-150 (2016).

16. Paniccia, M., Paniccia, D., Thomas, S., Taha, T., Reed, N. Clinical and non-clinical depression and anxiety in young people: A scoping review on heart rate variability. Autonomic Neuroscience. (2017).

17. Ellis, M.J., Leddy, J., Willer, B. Multi-Disciplinary Management of Athletes with Post-Concussion Syndrome: An Evolving Pathophysiological Approach. Frontiers in Neurology. 7 (2016).

18. Leddy, J., Baker, J.G., Haider, M.N., Hinds, A., Willer, B. A Physiological Approach to Prolonged Recovery From Sport-Related Concussion. Journal of Athletic Training. 52 (3), 299-308 (2017).

19. Senthinathan, A., Mainwaring, L.M., Hutchison, M. Heart Rate Variability of Athletes Across Concussion Recovery Milestones: A Preliminary Study. Clinical Journal of Sport Medicine. 27 (3), 288-295 (2017).

20. Kleiger, R.E., Stein, P.K., Bigger, J.T. Heart rate variability: measurement and clinical utility. Annals of Noninvasive Electrocardiology: The Official Journal of the International Society for Holter and Noninvasive Electrocardiology, Inc. 10 (1), 88-101 (2005).

21. Biswas, A.K., Scott, W.A., Sommerauer, J.F., Luckett, P.M. Heart rate variability after acute traumatic brain injury in children. Critical Care Medicine. 28 (12), 3907-3912 (2000).

22. Eckberg, D.L. Parasympathetic cardiovascular control in human disease: a critical review of methods and results. The American Journal of Physiology. 239 (5), H581-593 (1980).

23. Godin, G., Shepard, R.. Godin leisure-time Exercise questionnaire. Medicine \& Science in Sports \& Exercise. (29), S36-s38 (1997).

24. Sady, M.D., Vaughan, C.G., Gioia, G.A. Psychometric characteristics of the postconcussion symptom inventory in children and adolescents. Archives of Clinical Neuropsychology: The Official Journal of the National Academy of Neuropsychologists. 29 (4), $348-363$ (2014).

25. Moser, R.S., Schatz, P., Grosner, E., Kollias, K. One year test-retest reliability of neurocognitive baseline scores in 10- to 12-year olds. Applied Neuropsychology: Child. 6 (2), 166-171 (2017).

26. Paniccia, M. et al. Heart rate variability in non-concussed youth athletes: Exploring the effect of age, sex and concussion-like symptoms. Frontiers in Neurology. 8, 753 (2018).

27. Goldstein, D.S., Bentho, O., Park, M.-Y., Sharabi, Y. Low-frequency power of heart rate variability is not a measure of cardiac sympathetic tone but may be a measure of modulation of cardiac autonomic outflows by baroreflexes: Low-frequency power of heart rate variability. Experimental Physiology. 96 (12), 1255-1261 (2011).

28. Billman, G.E. The LF/HF ratio does not accurately measure cardiac sympatho-vagal balance. Frontiers in Physiology. 4 (2013).

29. Reed, N. et al. A Multi-Modal Approach to Assessing Recovery in Youth Athletes Following Concussion. Journal of Visualized Experiments. (91) (2014).

30. Paniccia, M.J., Reed, N.P. Dove and hawk profiles in youth concussion: Rethinking occupational performance: Voir le rendement occupationnel sous un angle différent en utilisant les profils de la « colombe » et du « faucon » pour aborder les commotions chez les jeunes. Canadian Journal of Occupational Therapy. (2017).

31. Reed, N. Sport-Related Concussion and Occupational Therapy: Expanding the Scope of Practice. Physical \& Occupational Therapy in Pediatrics. 31 (3), 222-224 (2011).

32. Carson, J.D. et al. Premature return to play and return to learn after a sport-related concussion: physician's chart review. Canadian Family Physician Medecin De Famille Canadien. 60 (6), e310, e312-315 (2014).

33. Leddy, J.J., Kozlowski, K., Donnelly, J.P., Pendergast, D.R., Epstein, L.H., Willer, B. A Preliminary Study of Subsymptom Threshold Exercise Training for Refractory Post-Concussion Syndrome. Clinical Journal of Sport Medicine. 20 (1), 21-27 (2010).

34. Friedman, B.H. An autonomic flexibility-neurovisceral integration model of anxiety and cardiac vagal tone. Biological Psychology. 74 (2), 185-199 (2007).

35. Friedman, B.H., Thayer, J.F. Anxiety and autonomic flexibility: a cardiovascular approach. Biological Psychology. 49 (3), $303-323$ (1998).

36. Kamins, J. et al. What is the physiological time to recovery after concussion? A systematic review. British Journal of Sports Medicine. $\mathbf{5 1}$ (12), 935-940 (2017).

37. Bornas, X., Balle, M., De la Torre-Luque, A., Fiol-Veny, A., Llabrés, J. Ecological assessment of heart rate complexity: Differences between high- and low-anxious adolescents. International Journal of Psychophysiology. 98 (1), 112-118 (2015).

38. Buchheit, M., Platat, C., Oujaa, M., Simon, C. Habitual Physical Activity, Physical Fitness and Heart Rate Variability in Preadolescents. International Journal of Sports Medicine. 28 (3), 204-210 (2007). 
39. Gutin, B., Howe, C., Johnson, M.H., Humphries, M.C., Snieder, H., Barbeau, P. Heart rate variability in adolescents: relations to physical activity, fitness, and adiposity. Medicine and Science in Sports and Exercise. 37 (11), 1856-1863 (2005).

40. Nagai, N., Moritani, T. Effect of physical activity on autonomic nervous system function in lean and obese children. International Journal of Obesity. 28 (1), 27-33 (2003).

41. Farah, B.Q., Barros, M.V.G., Balagopal, B., Ritti-Dias, R.M. Heart Rate Variability and Cardiovascular Risk Factors in Adolescent Boys. The Journal of Pediatrics. 165 (5), 945-950 (2014).

42. Hunt, A.W., Mah, K., Reed, N., Engel, L., Keightley, M. Oculomotor-Based Vision Assessment in Mild Traumatic Brain Injury: A Systematic Review. The Journal of Head Trauma Rehabilitation. (2015).

43. Abaji, J.P., Curnier, D., Moore, R.D., Ellemberg, D. Persisting Effects of Concussion on Heart Rate Variability during Physical Exertion. Journal of Neurotrauma. 33 (9), 811-817 (2016).

44. Gall, B., Parkhouse, W., Goodman, D. Heart rate variability of recently concussed athletes at rest and exercise. Medicine and Science in Sports and Exercise. 36 (8), 1269-1274 (2004).

45. Voss, A., Schroeder, R., Heitmann, A., Peters, A., Perz, S. Short-Term Heart Rate Variability-Influence of Gender and Age in Healthy Subjects. PLOS One. 10 (3), e0118308 (2015).

46. Kim, H.-S., Yoon, K.-H., Cho, J.-H. Diurnal Heart Rate Variability Fluctuations in Normal Volunteers. Journal of Diabetes Science and Technology. 8 (2), 431-433 (2014)

47. Saeki, Y., Atogami, F., Takahashi, K., Yoshizawa, T. Reflex control of autonomic function induced by posture change during the menstrual cycle. Journal of the Autonomic Nervous System. 66 (1-2), 69-74 (1997).

48. Sato, N., Miyake, S., Akatsu, J., Kumashiro, M. Power spectral analysis of heart rate variability in healthy young women during the normal menstrual cycle. Psychosomatic Medicine. 57 (4), 331-335 (1995). 